\title{
Philosophiques
}

\section{Le républicanisme, une solution de rechange au libéralisme politique?}

\section{Ophélie Desmons}

Volume 46, numéro 1, printemps 2019

La démocratie entre substance et procédure

URI : https://id.erudit.org/iderudit/1062014ar

DOI : https://doi.org/10.7202/1062014ar

Aller au sommaire du numéro

\section{Éditeur(s)}

Société de philosophie du Québec

ISSN

0316-2923 (imprimé)

1492-1391 (numérique)

Découvrir la revue

Citer cet article

Desmons, O. (2019). Le républicanisme, une solution de rechange au libéralisme politique? Philosophiques, 46(1), 95-118.

https://doi.org/10.7202/1062014ar
Résumé de l'article

Comment interpréter le renouveau du républicanisme ? Faut-il y voir, comme c'est souvent le cas, une tentative de formuler une véritable option de remplacement au libéralisme politique ? Le républicanisme est-il une théorie parfaitement distincte du libéralisme politique, capable de ne pas sombrer dans les mêmes écueils ? L'ambition de cet article est d'apporter des éléments de réponse à ces questions, en se focalisant sur le cas de la liberté de conscience. En faisant dialoguer le libéralisme politique de Rawls et le républicanisme critique de Cécile Laborde, j'entends montrer comment le républicanisme s'emploie à dépasser le libéralisme politique. Je souligne néanmoins les difficultés d'une interprétation perfectionniste du républicanisme et lui oppose une interprétation neutraliste. Cette interprétation neutraliste remet cependant en question l'idée selon laquelle le républicanisme est une option de rechange au libéralisme politique. 


\title{
Le républicanisme, une solution de rechange au libéralisme politique?
}

\author{
OPHÉLIE DESMONS
}

Maitre de conférences, ESPE de Paris, Sorbonne - Université, laboratoire

Sciences, Normes, Démocratie — UMR 7107.

\begin{abstract}
RÉSUMÉ. - Comment interpréter le renouveau du républicanisme? Faut-il y voir, comme c'est souvent le cas, une tentative de formuler une véritable option de remplacement au libéralisme politique? Le républicanisme est-il une théorie parfaitement distincte du libéralisme politique, capable de ne pas sombrer dans les mêmes écueils? L'ambition de cet article est d'apporter des éléments de réponse à ces questions, en se focalisant sur le cas de la liberté de conscience. En faisant dialoguer le libéralisme politique de Rawls et le républicanisme critique de Cécile Laborde, j'entends montrer comment le républicanisme s'emploie à dépasser le libéralisme politique. Je souligne néanmoins les difficultés d'une interprétation perfectionniste du républicanisme et lui oppose une interprétation neutraliste. Cette interprétation neutraliste remet cependant en question l'idée selon laquelle le républicanisme est une option de rechange au libéralisme politique.
\end{abstract}

ABSTRACT. - What does the renewal of republicanism mean? Are we, as a lot of people think, to understand republicanism as a real alternative to political liberalism? Is republicanism perfectly distinct from political liberal? Is it able to avoid its pitfall? This paper addresses these questions, focusing on the case of freedom of conscience. Through a dialogue between Rawls's political liberalism and Cécile Laborde's critical republicanism, I explain why republicanism claims it overcomes political liberalism. Nevertheless, I emphasise on the fact that a perfectionist interpretation of republicanism presents some difficulties and I propose an alternative neutralist interpretation. However, such an interpretation calls into question the idea that republicanism is an alternative to political liberalism.

\section{Introduction}

Dans le champ de la philosophie politique, l'un des événements manquants de la fin du vingtième siècle et du début du vingt et unième siècle, c'est la redécouverte et le renouvellement du républicanisme ${ }^{1}$. Cette redécouverte et ce renouvellement se sont opérés tant sur le plan de l'histoire des idées, notamment avec les travaux de Quentin Skinner et de l'école de Cambridge²,

1. Audier, Les Théories de la république, p. 7I ; Renaut, Qu'est-ce qu'un peuple libre?

2. Skinner, Machiavel; Skinner, La liberté avant le libéralisme; Skinner, Hobbes et la conception républicaine de la liberté; Dunn, La pensée politique de John Locke; Pocock, Le moment machiavélien, La pensée politique florentine et la tradition républicaine atlantique; Pocock, Political Thought and History. 
que sur le plan de la philosophie normative, notamment avec Philip Pettit et Cécile Laborde 3 .

Les positions développées et soutenues par les partisans du républicanisme présentent bien des différences. Il semble néanmoins que l'une de leurs motivations et l'un de leurs horizons communs soient de proposer une option de rechange au libéralisme politique. Ces penseurs considèrent que le libéralisme politique d'inspiration rawlsienne qui est devenu hégémonique présente un certain nombre de défauts importants et qu'il est nécessaire de faire émerger une nouvelle voie, qui permettra de sortir des impasses du libéralisme politique. Ils pensent qu'en revisitant et en amendant la tradition républicaine, ils peuvent faire émerger cette autre option. Ainsi, dans Français encore un effort pour être républicains!, Cécile Laborde écrit: "Le républicanisme à la française, s'il est réinterprété à la lumière de la critique sociale de la nondomination, peut constituer une alternative séduisante aux théories libérales et multiculturalistes qui dominent la pensée anglo-américaine ${ }^{4}$.»

Mais le républicanisme est-il à la hauteur de ses ambitions? Le républicanisme revisité parvient-il à dépasser le libéralisme politique ? Et le républicanisme doit-il effectivement être considéré comme une solution de rechange au libéralisme politique?

L'ambition de cet article est d'apporter des éléments de réponse à ces questions en partant d'un cas particulier, celui de la liberté de conscience. Mon intention est de montrer qu'un républicanisme critique comme celui de Cécile Laborde semble bien de prime abord présenter un certain nombre d'avantages par rapport à un libéralisme politique d'inspiration rawlsienne. Une perspective républicaine, notamment parce qu'elle accorde à la question de l'éducation une place centrale, semble plus à même de penser les conditions d'une liberté de conscience réelle et non simplement formelle. Je soutiendrai néanmoins que, tant que le républicanisme est conçu comme une solution de rechange au libéralisme politique, il fait face à une difficulté théorique majeure: le problème du perfectionnisme. Sortir de cette difficulté n'est pas impossible, et un certain nombre de républicains, comme Laborde, prennent à mon avis ce chemin. Mais alors, le républicanisme ne peut plus être conçu comme une solution de rechange au libéralisme politique. Il est simplement une variante du libéralisme politique.

3. Pettit, Républicanisme; Pettit, On the People's Term; Laborde, Critical Republicanism; Laborde, Français, encore un effort pour être républicains!; Laborde, Liberalism's Religion; Spitz, Le moment républicain en France; Spitz, Philip Pettit. À ces penseurs explicitement républicains, on pourrait en ajouter d'autres, comme Michaël Sandel ou Charles Taylor, qu'on classe plus souvent parmi les "communautariens » et dont le rapport au républicanisme mériterait d'être discuté. Sandel, "La république procédurale et le moi désengagé »; Sandel, Le Libéralisme et les limites de la justice; Taylor, Malaise de la modernité; Taylor, "Quiproquos et malentendus: le débat communautariens-libéraux "; Taylor, Multiculturalisme.

4. Laborde, Français, encore un effort pour être républicains!, p. 8. Je souligne. 
Dans une première section, je proposerai une première analyse comparative du libéralisme politique et du républicanisme afin de clarifier les termes de la critique républicaine. Dans les sections deux et trois, j'analyserai les forces et les faiblesses de chaque position au regard de la question de la liberté de conscience. Dans une quatrième section, je tenterai de procéder à un arbitrage, qui me conduira à compléter mon analyse du libéralisme politique, puis à confronter deux interprétations du républicanisme: une interprétation perfectionniste et une interprétation neutraliste.

\section{Libéralisme politique et républicanisme. Une première analyse comparative}

Pourquoi les républicains pensent-ils qu'il est nécessaire de construire une alternative au libéralisme politique devenu hégémonique? Pour apporter des éléments de réponse à cette question, précisons d'abord les traits caractéristiques du libéralisme politique et du républicanisme. Les couples procédure/substance et neutralisme/perfectionnisme sont à ce titre fort utiles.

\subsection{Le libéralisme politique: neutralisme et procédure}

Les principaux architectes du libéralisme politique contemporain ont très souvent affirmé que la caractéristique qui fait le propre du libéralisme, c'est la neutralités. L'État libéral, disent-ils, doit être neutre: il doit se garder d'imposer ou de promouvoir une conception particulière du monde ou du bien.

Mais pourquoi l'État libéral devrait-il être neutre? Pour répondre à cette question, les libéraux recourent souvent à une justification substantielle, en faisant appel aux valeurs qui se trouvent au fondement de l'État libéral: la liberté et l'égalité. Par définition, disent-ils, un État libéral s'engage à considérer les personnes comme libres et égales entre elles. Or, pour ce faire, il doit nécessairement être neutre. L'État doit s'abstenir d'imposer ou de promouvoir une conception particulière du monde et du bien afin que les personnes soient véritablement libres de choisir celle qui leur convient et afin qu'elles reçoivent le même traitement, quel que soit leur choix ${ }^{6}$. Si l'État impose ou promeut un contenu doctrinaire particulier, qu'il soit religieux ou séculier, la liberté et l'égalité des personnes sont compromises.

C'est néanmoins une approche procédurale qui permet de comprendre le véritable fondement de l'exigence de neutralité. Dans Libéralisme politique, Rawls explique que tout État exerce une forme de coercition. Les lois formulent en effet des interdictions. Dans le cadre d'un État libéral qui s'engage à respecter la liberté des citoyens, la question se pose de savoir à quelle condition cette coercition peut être justifiée. Rawls explique que l'État

5. Dworkin, "Le Libéralisme», in Berten, Da Silveira, Pourtois, Libéraux et communautariens, p. 5I-86; Larmore, Patterns of Moral Complexity; Larmore, Modernité et morale; Barry, Justice as Impartiality; Rawls, Théorie de la justice; Rawls, Libéralisme politique.

6. Pour ces arguments, Dworkin, «Le Libéralisme»; Rawls, Libéralisme politique. 
libéral se doit de respecter le "principe libéral de légitimité », selon lequel "notre exercice du pouvoir politique n'est correct et ne peut donc être justifié que lorsqu'il s'accorde avec une Constitution dont on peut raisonnablement espérer que tous les citoyens souscriront à ses exigences essentielles, à la lumière de principes et d'idéaux acceptables pour eux en tant qu'ils sont raisonnables et rationnels ${ }^{7}$. Pour le dire autrement, pour qu'une loi puisse être tenue pour légitime, les citoyens doivent pouvoir se considérer comme ses auteurs. Or cela n'est possible que si la loi peut être justifiée dans des termes qu'ils peuvent reconnaitre comme leurs. La loi ne peut donc être justifiée en faisant appel à une doctrine controversée. Elle doit être justifiée de manière neutre. En ce sens, l'exigence de neutralité dérive de la procédure de légitimation de la loi démocratique.

\subsection{Le républicanisme: perfectionnisme et substance}

Par contraste à ce libéralisme politique neutraliste et procédural, le républicanisme peut être décrit comme une position perfectionniste et substantielle. Le républicanisme ne constitue pas un ensemble parfaitement unifié. On distingue notamment deux types de républicanisme - le républicanisme athénien et le républicanisme néo-romain — qui trouvent à s'incarner aussi bien chez des auteurs classiques que chez des penseurs contemporains ${ }^{8}$. Du côté du républicanisme athénien, on trouvera notamment Aristote, Montesquieu, Rousseau ou Hannah Arendt. Du côté du républicanisme néo-romain, Machiavel ou Tocqueville.

Ces républicains ont néanmoins certainement un point commun. Ils assument une forme de perfectionnisme. Ils pensent que l'État ne doit pas être neutre et qu'il doit au contraire promouvoir certaines valeurs et vertus substantielles. Le républicanisme repose en particulier sur une valorisation des vertus de la citoyenneté et promeut la participation active à la vie politique.

Chaque type de républicanisme a cependant sa manière et ses raisons propres de valoriser la participation à la vie politique. Dans le républicanisme athénien, la participation est une fin en soi. Elle est conçue comme étant en elle-même un bien. Par exemple, pour Aristote, la participation à la vie de la cité est, pour l'homme, l'une des façons privilégiées de réaliser sa nature spécifique. Par l'engagement citoyen, l'homme développe et réalise son intellect, cette partie de l'âme qui le distingue de l'animal. Rousseau partage en un sens cette perspective. Seul le citoyen, capable d'entendre la volonté générale qui est en lui, par-delà ses intérêts particuliers et immédiats ${ }^{9}$, accède au stade ultime de la liberté, la liberté morale «qui seule rend l'homme véritablement maître de lui-même ${ }^{10}$ ».

7. Rawls, Libéralisme politique, p. 264.

8. Audier, Les Théories de la république.

9. Rousseau, Du contrat social, p. 59-60.

10. Ibid., p. 6I. 
Dans le républicanisme néo-romain, la participation politique a au contraire une valeur instrumentale. Ainsi, si Tocqueville en vient à soutenir qu'il faut encourager la participation aux affaires publiques, c'est parce que, selon lui, le désengagement aboutit à la destruction de la liberté. Pour préserver les libertés individuelles, il faut que les individus consacrent une partie de leur énergie à la chose publique.

Dans les deux cas, néanmoins, la neutralité des libéraux leur est reprochée. Elle est jugée indésirable par les partisans du républicanisme athénien, qui estiment que la nature humaine ne peut s'y accomplir. Elle est jugée insuffisante par les républicains néo-romains, qui soulignent qu'elle conduit à un despotisme doux, à la destruction de la liberté et finalement à l'instabilité du régime démocratique ${ }^{11}$. Selon les républicains, l'engagement des libéraux vis-à-vis de la neutralité rend leur théorie politique vulnérable et instable. Le républicanisme qui, au contraire, assume une forme de perfectionnisme, peut quant à lui faire la promotion des vertus citoyennes. Il peut dès lors parer au problème du délitement du lien social et contrecarrer le désengagement politique. Ce faisant, il constitue une solution de rechange attractive au libéralisme politique.

\subsection{Néo-républicanisme et perfectionnisme: un rapport complexe}

Les choses se complexifient néanmoins lorsque l'on se tourne vers le type de républicanisme qu'ont développé ces deux dernières décennies des auteurs comme Philip Pettit et Cécile Laborde. Leur rapport au perfectionnisme est en effet plus complexe.

Par exemple, dans Français encore un effort pour être républicains!, Cécile Laborde semble bien assumer une forme de perfectionnisme. Elle affirme qu'elle s'inspire de Philip Pettit tout en soulignant que «Pettit a défendu l'idée que la théorie républicaine bien comprise promouvait une valeur particulière: celle de la non-domination ${ }^{12}{ }^{\prime}$. Laborde, comme Pettit, seraient perfectionnistes, au sens où ils défendraient la promotion d'une valeur substantielle particulière: la liberté comme non-domination ${ }^{13}$.

Néanmoins, comme on le verra, Laborde n'est pas prête à accepter n'importe quel type de perfectionnisme. Comme Pettit, elle souhaite développer un républicanisme qui respecte le "fait du pluralisme raisonnable ", constaté et érigé en norme par les libéraux. Pettit rejette ainsi explicitement le républicanisme néo-aristotélicien adopté par Pocock. Il refuse de considérer qu'une vie bonne est nécessairement une vie vouée à la participation

11. Tocqueville, De la démocratie en Amérique, vol. 2, p. 385-386.

12. Laborde, Français, encore un effort pour être républicains!', p. 46.

13. Pettit, Républicanisme, p. I I : «si nous jugeons que l'État a pour finalité la promotion de la liberté comme non-domination [...], nous pourrons alors commencer de repérer les contours d'une philosophie politique convaincante». 
politique. Il appelle de ses vœux un républicanisme compatible avec une pluralité de conceptions de la vie bonne ${ }^{14}$.

Mais alors, ce républicanisme doit-il toujours être compris comme un perfectionnisme? Et continue-t-il d'être cette autre option qui permettrait d'échapper aux écueils du libéralisme politique?

Pour apporter des éléments de réponse à ces questions difficiles, je m'intéresserai, dans les deux sections suivantes, à un cas particulier: le cas de la liberté de conscience. Ce cas me semble constituer un angle d'attaque pertinent puisqu'il permet de mieux saisir les défauts qui peuvent, à première vue, être ceux d'une perspective libérale et les avantages comparatifs d'une perspective républicaine.

\section{La liberté de conscience dans le libéralisme politique}

Comment un libéral aborde-t-il la question de la liberté de conscience? Plus précisément, qu'est-ce qui, pour un libéral, est nécessaire à la mise en œuvre de la liberté de conscience ? Et quels sont les éventuels défauts de cette perspective? Pour répondre à ces questions, je m'appuierai, dans cette section, sur les réponses développées par John Rawls qui, bien qu'elles ne soient plus récentes, demeurent robustes.

\subsection{John Rawls, la liberté de conscience comme première signification de la liberté}

Rawls définit les personnes comme libres et égales entre elles, rationnelles et raisonnables. Mais Rawls n'en reste pas à cette définition générale. Il s'emploie à préciser le sens de ces termes et distingue notamment trois significations de la liberté ${ }^{15}$. C'est la première qui nous intéresse: "premièrement, les citoyens sont libres en ce sens qu'ils considèrent qu'eux-mêmes, comme tout autre, possèdent la faculté morale de former une conception du bien ${ }^{16}{ }^{\prime}$. La première signification de la liberté, c'est, pour Rawls, la liberté de conscience. La liberté de conscience est d'abord un fait. C'est le fait que les personnes soient capables de former une conception de la vie bonne, parce qu'elles possèdent une faculté qui leur permet de choisir la conception de la vie bonne qui leur convient. Mais Rawls ajoute que:

En tant que citoyens, ils sont plutôt conçus comme capables de réviser et de changer cette conception en se fondant sur des arguments raisonnables et rationnels. [...] Étant donné leur faculté morale de former, de réviser et de poursuivre rationnellement une conception du bien, leur identité publique de personnes libres n'est pas affectée par les changements dans le temps de leur conception précise de ce bien ${ }^{17}$.

14. Pettit, Républicanisme, p. 26.

15. Rawls, Libéralisme politique, p. 55-6I ; Rawls, La justice comme équité, p. 42-46.

16. Ibid., p. 55. On trouve une formulation équivalente dans Rawls, La justice comme équité, p. 43.

17. Rawls, Libéralisme politique, p. 55-56. 
La liberté de conscience est donc surtout un droit. Les personnes sont libres parce que, dans le cadre de la démocratie constitutionnelle, leur «identité publique ", c'est-à-dire leur citoyenneté, est déconnectée de la doctrine compréhensive particulière à laquelle elles adhèrent. Leur statut de citoyen n'est pas conditionné au fait d'adhérer à telle doctrine compréhensive, par exemple à une religion d'État. Pour le dire autrement, les personnes disposent d'un droit de sortie. Elles peuvent changer de doctrine compréhensive sans que ce changement n'affecte leur statut politique.

Finalement, et contrairement à ce que certains communautariens ont suggéré18, Rawls n'affirme pas que les personnes sont libres au sens où elles choisiraient leur conception de la vie bonne indépendamment de toute forme de détermination sociologique ou d'attachement préalable. Une telle conception serait tout à fait irréaliste. Reste que le fait que nous soyons sociologiquement déterminés ne change rien au fait que nous soyons politiquement libres. Nous avons la possibilité de changer de conception de la vie bonne sans que cela n'affecte nos droits en tant que citoyens.

Cette interprétation libérale de la liberté pose en revanche une autre question: même si l'on admet que la liberté de conscience dépend d'un droit de sortie et non d'un fait sociologique, la question se pose de savoir comment faire pour s'assurer que notre liberté de conscience est bien réelle et non simplement formelle. Il est en effet possible que tout en possédant, d'un point de vue juridique, un droit de sortie, je ne puisse effectivement jouir de ce droit.

Sur la route qui mène à la sortie d'une doctrine compréhensive, les obstacles sont nombreux. L'un de ces obstacles est de nature psychologique. Si l'ensemble de ma vie sociale, depuis ma naissance, s'opère dans une seule et même communauté culturellement homogène, la doctrine compréhensive de ma communauté d'origine pourra m'apparaître comme la seule option imaginable. Cette doctrine compréhensive ne sera pas, pour moi, une option existentielle possible, parmi d'autres options existentielles. Elle s'imposera à moi sans que je ne puisse la mettre à distance. Si, à aucun moment, je ne suis capable de me demander si je veux continuer d'adhérer à cette doctrine, mon droit de sortie n'est-il pas parfaitement formel et finalement illusoire? Comment dès lors s'assurer que, dans une démocratie constitutionnelle, la liberté de conscience des citoyens soit bien réelle?

\subsection{Les conditions libérales d'une liberté de conscience réelle}

Rawls apporte des éléments de réponse à cette question. Notons tout d'abord que, de manière générale, Rawls ne se satisfait pas de droits formels. Il juge notamment que l'égalité formelle des chances (le «système de la

18. Sandel, «La république procédurale et le moi désengagé »; Sandel, Le Libéralisme et les limites de la justice. 
liberté naturelle ${ }^{19}$ ») est insuffisante et lui préfère le principe d'égalité équitable des chances. Il considère que l'ouverture légale des positions sociales est insuffisante et qu'il faut chercher à contrecarrer les phénomènes de reproduction sociale. À dons égaux et à volonté égale de développer nos dons, nous devrions avoir les mêmes probabilités de parvenir aux positions que nous convoitons, quelle que soit notre position sociale initiale.

Que dit-il de la liberté de conscience? Dans la cinquième leçon de Libéralisme politique, Rawls s'intéresse à la question de l'éducation des enfants qui grandissent dans des «sectes religieuses [qui] s'opposent à la culture du monde moderne et souhaitent mener leur vie commune à l'écart de ses effets indésirables ${ }^{20}$ ». Il s'agit là d'une allusion à l'affaire Wisconsin $v$. Yoder. En 1968, les parents de trois adolescents issus de la communauté Amish interrompent la scolarité obligatoire de leurs enfants, arguant du fait qu'il est essentiel, qu'au-delà de l'âge de quatorze ans, les enfants ne soient plus éloignés de la communauté, sans quoi il deviendrait très difficile de leur transmettre la foi et le mode de vie de la communautée ${ }^{21}$. La question que pose Rawls est donc la suivante: comment s'assurer que ces jeunes soient effectivement libres de rompre avec les croyances dans lesquelles ils ont été élevés? Comment s'assurer que leur liberté de conscience est réelle?

La réponse qu'il propose se distingue par sa modestie assumée. Rawls explique que les exigences du libéralisme politique sont, en la matière, inférieures à celles du libéralisme compréhensif de Mill ou de Kant, qui accordent à l'individualité et à l'autonomie une valeur intrinsèque:

Il [le libéralisme politique] demandera simplement que l'enseignement comporte l'idée de droits civiques et constitutionnels des jeunes afin qu'ils sachent que la liberté de conscience existe dans leur société et que l'apostasie n'est pas un crime aux yeux de la loi, tout cela afin de garantir que, lorsqu'ils deviendront adultes, leur adhésion à cette secte religieuse ne sera pas basée sur l'ignorance de leurs droits fondamentaux ou sur la peur de châtiments pour des crimes qui n'existent pas $^{22}$.

Rawls donne ici quelques indications de ce que pourrait être une politique scolaire émanant de la théorie de la justice comme équité. Garantir une liberté de conscience réelle, c'est la mission de l'école. Cette mission est accomplie tant que l'enseignement dispensé permet aux jeunes de savoir que le droit positif leur garantit la liberté de conscience. Les jeunes doivent apprendre à l'école qu'ils peuvent sans crainte renoncer à la foi dans laquelle ils ont été élevés, et que cette rupture sera sans conséquence sur leur statut de citoyen.

19. Rawls, Théorie de la justice, p. I03.

20. Rawls, Libéralisme politique, p. 243. Le terme «secte» n'a pas ici de sens péjoratif.

21. Pour un exposé plus complet, Dilhac, La tolérance, un risque pour la démocratie?, p. I 5 I-I99.

22. Rawls, Libéralisme politique, p. 243-244. Je souligne. 
Finalement, ce qui semble caractériser cette conception libérale de l'éducation à la liberté de conscience, c'est une forme d'intellectualisme. Si on l'adopte, on devra admettre que les jeunes Amish sont réellement libres à condition (i) qu'ils disposent d'un droit de sortie, ce qui est toujours le cas dans une démocratie constitutionnelle; et (ii) qu'ils sachent qu'ils disposent de ce droit, ce qui sera le cas si on le leur a bien dit à l'école. La connaissance du droit semble, du point de vue libéral, suffire à rendre la liberté de conscience réelle.

Rawls reconnaît volontiers le caractère limité de la conception qu'il soutient. Il justifie néanmoins cette restriction:

La théorie de la justice comme équité ne cherche pas à cultiver les vertus et les valeurs propres au libéralisme, à savoir l'autonomie et l'individualité, pas plus d'ailleurs que celles d'autres doctrines compréhensives. Car, autrement, elle cesserait d'être une forme du libéralisme politique ${ }^{23}$.

Il n'y a pas lieu, rappelle Rawls, d'encourager les individus à rompre avec la religion. La théorie libérale doit en effet être neutre à l'égard des différentes doctrines compréhensives raisonnables. Elle ne doit en promouvoir aucune, sans quoi elle bafoue ses engagements vis-à-vis de l'égale liberté des personnes.

Mais cette conception de la liberté de conscience est-elle satisfaisante? Plus précisément, est-elle satisfaisante du point de vue des défenseurs du républicanisme?

\section{La liberté de conscience dans le républicanisme}

Certains républicains dénoncent l'insuffisance de cette conception et affirment que le républicanisme permet une approche plus satisfaisante de la liberté de conscience. C'est à mon avis le cas de Cécile Laborde qui a développé un "républicanisme critique" que j'estime robuste dans Français, encore un effort pour être républicains! $!^{24}$

Dans ce livre, avant de développer sa propre position, Laborde reconstruit un républicanisme qu'elle nomme «classique » et qui correspond, selon elle, au républicanisme orthodoxe issu de la troisième république, républicanisme qui possède toujours une force d'influence notable, notamment en France $^{25}$. En m'appuyant sur la reconstruction qu'en propose Laborde, je montrerai pourquoi les partisans du républicanisme classique jugent la conception libérale de la liberté de conscience insuffisante, et exposerai la conception de rechange qu'ils soutiennent. Je m'arrêterai ensuite sur les

23. Rawls, Libéralisme politique, p. 244.

24. Ce livre est l'adaptation en français d'un ouvrage d'abord écrit en anglais, Laborde, Critical Republicanism, auquel je me référerai également.

25. Ce républicanisme partage certains de ses traits essentiels avec ce que Jean Baubérot appelle la laïcité gallicane. Baubérot, Les sept laïcités françaises. 
avantages et les difficultés de cette position, et en particulier sur les difficultés que pose son perfectionnisme spécifique. J'indiquerai enfin comment le républicanisme critique que Laborde développe entend dépasser ces difficultés tout en échappant aux écueils du libéralisme.

\subsection{Républicanisme classique et liberté de conscience}

Les partisans du républicanisme classique récusent la conception libérale de la liberté de conscience. Selon eux, contrairement à ce que croient les libéraux, être libre, ce n'est pas avoir la possibilité d'adhérer à n'importe quel type de croyance:

Certaines formes de croyance - celles qui impliquent l'abdication de la capacité humaine à l'autodétermination rationnelle et autonome - sont incompatibles avec la pensée libre et la citoyenneté éclairée ${ }^{26}$.

Pour les républicains classiques, être libre, c'est être capable de penser librement. C'est être capable d'un "exercice actif de la raison ${ }^{27}$ ». Un être libre ne pourra donc accepter des positions qui supposent l'abdication de la raison. Il ne pourra accepter de croire, c'est-à-dire de tenir pour vraies des propositions dont il est par définition impossible de démontrer qu'elles le sont. Il ne pourra accepter le dogmatisme que l'attitude religieuse réclame.

Ainsi, les républicains classiques rejettent la conception libérale de la liberté de conscience au profit d'une autre conception de la liberté. Ils considèrent que la liberté de pensée doit toujours l'emporter sur la liberté de conscience. Plus précisément, ils considèrent que la liberté de conscience ne peut être comprise autrement que comme une liberté de pensée ${ }^{28}$.

Les républicains classiques assument donc une première forme de perfectionnisme éthique. Leur position est fondée sur l'idée selon laquelle certains modes de vie ont plus de valeur que d'autres. Selon eux, une vie de croyance ne peut pas être une vie bonne. Pour vivre une vie bonne, il faut nécessairement rompre avec l'attitude religieuse de renonciation à l'usage de la raison. Il faut vivre sa vie "de l'intérieur ${ }^{29}$ ", c'est-à-dire vivre une vie de «remise en cause critique des croyances, préjugés et tradition $s^{30}$ ».

De ce perfectionnisme éthique découle un perfectionnisme politique. Les républicains classiques soutiennent un certain type d'agencement institutionnel qui, selon eux, permettra de réaliser cette liberté de conscience

26. Laborde, Français, encore un effort pour être républicains!, p. 24.

27. Ibid., p. 23.

28. Sur cette conception de la liberté de conscience comme liberté de pensée, Laborde renvoie à Baubérot, La morale lä̈que contre l'ordre moral, p. 305-3 I4; Baubérot, "La laïcité comme pacte laïque", p. 47-49. On se reportera aussi à Baubérot, Les sept laïcités françaises, p. 46-47.

29. Laborde, Critical Republicanism, p. I03. Je traduis.

30. Laborde, Français, encore un effort pour être républicains!, p. 23. 
entendue comme liberté de pensée. Cet agencement institutionnel repose sur deux piliers: l'État et l'école.

\section{(i) L'État}

À la différence des libéraux qui se méfient du pouvoir de l'État et entendent en limiter la portée, les républicains se démarquent par leur "confiance dans le pouvoir émancipateur de l'État ${ }^{31}{ }^{\prime}$. Ils estiment que le rôle de l'État républicain, c'est de «libér[er] les individus du joug des autorités traditionnelles — féodales, familiales, cléricales ${ }^{32} »$. L'État républicain doit aider les individus à se libérer de ces institutions qui entendent aliéner leur raison, en leur fournissant les moyens d'être autonomes.

Mais comment l'État républicain pourra-t-il libérer les individus? Les républicains classiques endossent un «paternalisme indirect ${ }^{33}$ » selon lequel c'est par l'intermédiaire de l'école que l'État républicain promeut sa conception de la liberté.

\section{(ii) L'école}

La «philosophie républicaine de l'éducation ${ }^{34}$ » estime que l'école républicaine ne transmet pas simplement des savoirs positifs. Son rôle est d'abord et avant tout d'offrir à chaque enfant les ressources et les outils nécessaires pour apprendre à faire usage de sa raison. Comme l'explique Catherine Kintzler ${ }^{35}$, qui puise ses sources d'inspiration chez Condorcet, "l'école est une institution productrice de liberté: on n'y vient pas pour consommer, ni même pour jouir de son droit, mais pour s'autoconstituer comme sujet ${ }^{36}$ ». L'école est ce lieu dans lequel les enfants acquièrent l'autonomie rationnelle nécessaire à l'exercice de la citoyenneté.

Mais, selon les républicains classiques, pour qu'un tel apprentissage soit possible, certaines conditions doivent être réunies. En premier lieu, "l'école n'est pas simplement un lieu d'apprentissage de l'autonomie; elle est aussi celui de l'expérience de l'autonomie ${ }^{37}$ ». Le républicanisme rejette le modèle intellectualiste qui était celui de l'éducation libérale. Pour apprendre à être libre, il ne suffit pas de connaître ses droits. Il faut pouvoir faire l'expérience de la liberté.

31. Ibid., p. 25.

32. Ibid., p. 25.

33. Laborde, Critical Republicanism, p. I I9. Je traduis.

34. Laborde, Français, encore un effort pour être républicains!, p. 29.

35. Kintzler, "Aux fondements de la laïcité scolaire. Essai de décomposition raisonnée du concept de laïcité »; Kintzler, La République en question. On pourra également se reporter à ses travaux plus récents: Kintzler, Qu'est-ce que la laïcité?; Kintzler, Penser la laïcité.

36. Kintzler, Qu'est-ce que la laïcité?, p. 55.

37. Laborde, Français, encore un effort pour être républicains!, p. 29. Cette idée d'expérience de la liberté est développée dans Kintzler, Qu'est-ce que la laïcité?, p. 56. 
Or, pour que cette expérience soit possible, un certain nombre de conditions doivent nécessairement être réunies: «il y a des conditions non spontanées de constitution de la libertée ${ }^{38}$ ». Concrètement, l'espace scolaire doit être neutralisé. Il doit notamment être expurgé des appartenances religieuses et communautaires. Il faut constituer un espace dans lequel les enfants "oublient leur communauté et pensent à autre chose qu'à ce qu'ils sont afin de penser par eux-mêmes ${ }^{39}$ ".

C'est la raison pour laquelle les républicains classiques, comme Kintzler, ont, en France, soutenu la loi de 2004 qui interdit aux élèves des écoles publiques le port de signes religieux ostensibles. Ils considèrent que l'école est un espace "à part» qui doit être considéré comme un "sanctuaire ». L'interdiction des signes religieux à l'école constitue, selon eux, la condition de possibilité de l'expérience de la libertée ${ }^{40}$. Les républicains classiques soutiennent ainsi cette mesure coercitive, considérant qu'elle est la condition de possibilité d'une constitution plus fondamentale de la liberté.

Faut-il accepter la critique du libéralisme qu'endossent les républicains classiques?

\subsection{Les difficultés du républicanisme classique: un perfectionnisme étroit et coercitif}

Laborde estime que le républicanisme classique pose plusieurs types de difficultés qui doivent nous en détourner. Elle affirme, premièrement, que son perfectionnisme éthique est trop étroit. À propos de la conception de la vie bonne que les républicains classiques soutiennent, Laborde écrit:

Les laïcistes se trompent quand ils érigent en valeur suprême et universelle l'autonomie en tant qu'émancipation de la religion et de la culture par la raison. [...] Une vie de piété, de dévouement aux autres, d'obéissance à une tradition n'est pas moins respectable ni moins humaine, si elle est librement acceptée, qu'une vie de désengagement existentiel. Les laïcistes ont donc tort de dire qu'une vie bonne est une vie où le sujet remet en permanence en cause ses engagements, ses croyances et ses liens affectifs. L'important est qu'il ait la capacité, le pouvoir de le faire, et qu'il puisse imaginer de le faire. Il faut, en somme, qu'il soit capable d'autonomie, sans que nécessairement il l'exerce dans sa vie quotidienne ${ }^{41}$.

Laborde admet que l'État promeuve certaines valeurs et vertus. Elle estime néanmoins que la conception de la vie bonne que les républicains classiques soutiennent est trop étroite. Ils ont tort, dit-elle, de considérer que seule une vie dans laquelle l'individu fait, en permanence, un usage actif de la raison est une vie bonne. Une vie de piété ou d'obéissance peut également

38. Kintzler, Qu'est-ce que la laïcité?, p. 55.

39. Laborde, Français, encore un effort pour être républicains!, p. 29. Il s'agit d'une citation de Kintzler.

40. Ibid., p. 30.

41. Ibid., p. 45-46. 
être une vie bonne. Selon elle, le républicanisme classique se trompe de cible. Ce qui importe, ce n'est pas que les individus rompent à chaque instant avec le dogme ou la tradition. Ce qui importe, ce n'est pas qu'ils exercent en permanence leurs facultés critiques, mais qu'ils soient capables de le faire. Ce qui importe, c'est que personne ne soit, d'une façon ou d'une autre, forcé de croire.

Dans Les religions face à l'intolérance, Martha Nussbaum formule un argument qui va dans le même sens. Elle affirme qu' «il est un fait qu'Américains et Européens doivent regarder en face: certaines personnes choisissent délibérément une vie placée sous le signe de l'autorité et de la contrainte $^{42}$ ». C'est, dit-elle, par exemple le cas de ceux qui choisissent d'embrasser une carrière militaire. Mais si nous admettons que la vie de ceux qui sacrifient leur autonomie à la défense de la patrie peut être une vie bonne, pourquoi le refuser à ceux qui adoptent une vie religieuse?

Laborde critique en outre le perfectionnisme politique coercitif de la loi de 2004. Ce perfectionnisme coercitif manque de cohérence. Selon Laborde, "l'interdiction coercitive est peu compatible avec la finalité déclarée de la $\operatorname{loi}^{43}$ ». Il y a, pense-t-elle, quelque chose de profondément paradoxal à prétendre libérer certaines personnes en faisant usage de la coercition. On prétend rendre possible l'expérience de la liberté en limitant la liberté.

Mais pourquoi alors ne pas simplement en revenir à une conception libérale?

\subsection{Républicanisme critique et liberté de conscience}

Malgré ces difficultés, Laborde considère qu'une perspective républicaine demeure nécessaire. Le libéralisme politique demeure en effet selon elle insuffisant, en particulier parce que les libéraux ne sont pas suffisamment méfiants vis-à-vis «des préférences, des croyances et des normes existantes $^{44}$ ». Ils ne sont pas assez attentifs aux conditions sociales dans lesquelles chacun forme ses préférences. Ils partent des préférences existantes, sans interroger leurs conditions de formation. Ils sont dès lors très souvent aveugles aux mécanismes de domination dont certaines personnes peuvent être victimes, domination d'autant plus subtile que les victimes elles-mêmes ne la perçoivent pas toujours "parce qu'elle s'accompagne d'une internalisation des normes ${ }^{45}$ ».

Pour revenir à l'exemple des jeunes Amish, Rawls semble considérer que le fait que ces jeunes gens savent (parce qu'ils l'ont appris à l'école) qu'ils ont le droit de quitter leur religion suffit pour considérer que leur pré-

42. Nussbaum, Les religions face à l'intolérance, p. I 80.

43. Laborde, Français, encore un effort pour être républicains!', p. 44.

44. Ibid., p. 49.

45. Ibid., p. 48. 
férence est le résultat d'un libre choix. Si la loi leur garantit un droit de sortie, qu'ils le savent et qu'ils continuent néanmoins d'adhérer à cette doctrine, c'est qu'ils le font librement. Mais, selon le républicanisme critique, cette conclusion est erronée. Connaître nos droits ne suffit pas. Si, en déscolarisant de manière précoce un adolescent, on le prive des facultés intellectuelles qui lui permettraient d'examiner de manière critique sa doctrine compréhensive d'origine, on abolit sa liberté de conscience. Si on le prive de cette capacité à formuler un jugement critique et à évaluer rétrospectivement le mode de vie dans lequel il a été élevé, on le rend incapable d'autonomie. On lui fait subir une forme de domination.

Laborde estime également que le républicanisme est mieux placé que le libéralisme politique pour surmonter cette difficulté. En effet, au contraire du libéralisme politique, astreint à la neutralité, le républicanisme est libre de promouvoir certaines valeurs particulières. Le républicanisme critique considère que l'État peut légitimement promouvoir cette valeur particulière qu'est la non-domination. L'État républicain fera la promotion d'une certaine conception de la liberté, conception qui se distingue aussi bien de la liberté négative des libéraux que de la liberté de pensée des républicains classiques. Il cherchera à s'assurer que les individus ne sont pas dominés. Il cherchera à les protéger "contre le pouvoir arbitraire d'autrui ${ }^{46}$ ».

Pour ce faire, «l'éducation à l'autonomie est essentielle ${ }^{47}$ ». À la différence des libéraux, les républicains sont attentifs au fait que notre faculté critique a, comme toute faculté, besoin d'être éduquée. Même si chacun dispose naturellement de cette faculté, elle doit être entraînée et exercée pour passer de la puissance à l'acte. Pour pouvoir faire usage de mon droit de sortie, il ne me suffit pas de savoir que je possède ce droit. Il me faut avoir appris à prendre du recul par rapport à mes options existentielles, à imaginer d'autres options et à faire des choix raisonnés. Il me faut avoir appris la pensée critique.

Mais quelles sont les conditions de possibilité d'un tel apprentissage? L'interdiction, pour les élèves, d'arborer des signes religieux dans les écoles publiques est-elle l'une de ses conditions, comme le pensent les républicains classiques? Laborde se positionne contre l'interdiction faite aux élèves des écoles publiques de porter des signes religieux par la loi de 2004. Plusieurs raisons fondent ce rejet. Premièrement, Laborde juge peu convaincant le recours à la coercition au nom de la promotion de l'autonomie. On l'a souligné plus haut, il y a en effet quelque chose de fondamentalement paradoxal à prétendre libérer des individus en réduisant leur liberté. Dans une perspective conséquentialiste, Laborde ajoute que «l'interdiction coercitive risque

46. Laborde, Français, encore un effort pour être républicains!, p. 47. Pour comprendre ce que Laborde entend par "non-domination ", on se reportera à Laborde, Français, encore un effort pour être républicains!, p. 46-5 I.

47. Ibid., p. 46. 
fort d'être contre-productive et d'exacerber l'affirmation défensive de normes et pratiques patriarcales ${ }^{48}$ ». Il est tout à fait possible que la coercition raidisse les rapports plutôt qu'elle ne les détende, qu'elle exclut plus qu'elle n'inclue ${ }^{49}$.

Mais surtout, Laborde estime que le débat sur le port des signes religieux à l'école a masqué une question plus fondamentale qu'il est, en France, devenu tout bonnement impossible d'aborder: la question de la légitimité du financement public des écoles privées à caractère religieux. Laborde rappelle que, selon l'idéologie républicaine officielle, «l'État a un devoir visà-vis de tous les enfants en tant que futurs citoyens: celui de les socialiser dans un espace commun qui soit "détaché" du milieu familial, et qui donne à tout enfant les mêmes opportunités ${ }^{50}$ ". Mais si l'éducation joue un rôle aussi fondamental dans la constitution de la citoyenneté, et si, comme l'affirme Kintzler, le détachement vis-à-vis du milieu familial est l'une des conditions de possibilité de l'expérience de la liberté, comment peut-on dans le même temps admettre qu'environ $20 \%$ des enfants soient scolarisés dans des écoles privées confessionnelles? Laborde estime que la commission Stasi, dont le rapport a abouti à la loi de 2004 et qui s'était également prononcée en faveur de la création d'écoles musulmanes, a omis de poser la question de savoir "si la séparation entre élèves que les écoles privées instituent et légitiment n'est pas une bien plus grave entorse à la laïcité et à la citoyenneté commune que le port du foulard dans l'enceinte de l'école

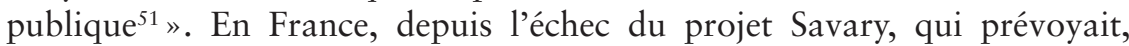
conformément au programme de François Mitterrand, la création d'un "grand service public unifié et laïque de l'éducation nationale", presque plus personne ne remet en cause le statut des écoles privées sous contrat. En outre, coïncidence ou non, c'est dans les années qui ont suivi l'abandon de ce projet que le débat s'est focalisé sur le port des signes religieux à l'école ${ }^{52}$. Laborde pose au contraire la question de savoir si le financement public d'écoles religieuses n'est pas une entorse bien plus sérieuse à la laïcité et au projet républicain que le port de signes religieux.

Venons-en maintenant à notre évaluation finale. Le républicanisme, dans la version révisée que Laborde développe, constitue-t-il en effet une solution de rechange solide et attractive au libéralisme politique ? Parvient-il à éviter les écueils dans lesquels le libéralisme politique semble condamné à tomber en raison de son neutralisme?

48. Ibid., p. 42-43.

49. C'est notamment ce qu'affirmait Locke dans la Lettre sur la tolérance: «Ce n'est pas la diversité des opinions, qu'on ne saurait éviter, mais le refus de la tolérance qu'on pourrait accorder, qui a été la source de toutes les guerres et de tous les démêlés qu'il y a eu parmi les chrétiens, sur le fait de la religion ", p. 2 I 2.

50. Laborde, Français, encore un effort pour être républicains!', p. 97.

51. Ibid., p. 96.

52. Les premières «affaires du foulard" remontent à I 989 . 


\section{Arbitrage: deux interprétations du républicanisme}

Pour pouvoir répondre à cette question, il me faut compléter ma présentation initiale du libéralisme politique. Une lecture approfondie révèle en effet que le libéralisme politique n'est pas si démuni que la critique républicaine le suggère.

\subsection{Libéralisme politique et promotion des valeurs et vertus politiques}

Les objections que les républicains adressent aux libéraux sont variées. Ainsi, si Tocqueville insiste sur la difficulté, dans un cadre libéral, de préserver la liberté et, finalement, la stabilité du régime démocratique ${ }^{53}$, Laborde reproche plutôt aux libéraux d'accepter les préférences des individus sans interroger les conditions de leur formation. Dans les deux cas, c'est l'engagement libéral à l'égard de la neutralité qui est en cause. Il empêcherait les libéraux de promouvoir la participation aux affaires publiques ou l'acquisition de l'esprit critique. En réalité, si on lit bien Rawls, on constate qu'il y a des raisons de douter que toutes ces critiques soient fondées. Dans Libéralisme politique, Rawls écrit:

Même si le libéralisme politique recherche un terrain commun et même s'il est neutre du point de vue du but, il est important de souligner que la possibilité d'affirmer la supériorité de certaines formes du caractère moral et d'encourager certaines vertus morales y est conservée. C'est pourquoi la théorie de la justice comme équité comprend une analyse de certaines vertus politiques - les vertus de la coopération sociale équitable telles que la civilité ou la tolérance, la modération ou le sens de l'équité. [...]

Les idées du bien peuvent être librement introduites, si nécessaire, pour compléter la conception politique de la justice; mais il faut que ce soit des idées politiques c'est-à-dire qu'elles appartiennent à une conception politique de la justice pour un régime constitutionnel. [...]

Si un régime constitutionnel prend certaines dispositions afin de renforcer les vertus de tolérance et de confiance mutuelle en s'opposant, par exemple, aux diverses formes de discrimination religieuse ou raciale (d'une façon compatible avec la liberté de conscience ou de parole), il ne devient pas pour autant un État perfectionniste au sens de Platon ou d'Aristote, et il n'établit pas non plus une religion particulière comme religion d'État comme dans le cas des États catholiques et protestants du début des Temps modernes. Il prend plutôt des mesures raisonnables pour renforcer les façons de penser et de sentir qui favorisent la coopération sociale entre des citoyens considérés comme libres et égaux, ce qui est tout à fait différent d'un État qui favoriserait une doctrine compréhensive particulière pour elle-même ${ }^{54}$.

53. On retrouve ce type d'argument dans Taylor, «Quiproquos et malentendus: le débat communautariens-libéraux".

54. Rawls, Libéralisme politique, p. 238-239. 
Rawls rappelle ici qu'il ne faut pas comprendre l'exigence libérale de neutralité comme une exigence de neutralité radicale. On l'a dit, l'État libéral s'engage à ne promouvoir aucune doctrine compréhensive raisonnable particulière. Mais cela ne l'engage pas à être radicalement neutre. Cela ne le condamne pas non plus à la passivité. L'État libéral reconnaît légitimement certaines valeurs : la liberté, l'égalité, l'équité. Il peut donc proscrire les actions qui contredisent les valeurs de liberté et d'égalité. Rawls considère en outre que l'État libéral a la possibilité de promouvoir activement certaines "vertus", qui, comme la coopération sociale, la civilité ou la tolérance, sont nécessaires à la réalisation de la liberté et de l'égalité. C'est la raison pour laquelle on voit le libéral qu'est Rawls emprunter le vocabulaire de la vertu, vocabulaire qui relève plutôt de la tradition républicaine. Selon Rawls, la promotion des vertus est légitime dans un cadre libéral tant que ce sont des vertus politiques. Elles pourront alors en effet être reconnues et adoptées par l'ensemble des citoyens, quelle que soit la doctrine compréhensive à laquelle ils adhèrent par ailleurs. Un État libéral peut donc par exemple faire la promotion de la vertu de "coopération sociale». Il peut, pour le dire autrement, encourager la participation politique, s'il considère qu'elle est un instrument indispensable à la réalisation de la liberté, qui est elle-même une valeur politique.

Un État libéral n'est pas non plus condamné à se cantonner à une éducation civique intellectualiste qui transmettrait de l'information juridique et permettrait à chacun de connaître ses droits. Rawls affirme qu'il peut légitimement chercher à renforcer «les façons de penser et de sentir qui favorisent la coopération sociale ${ }^{55} »$. L'éducation libérale peut légitimement chercher à produire des affects. Elle peut prendre les mesures qui conduiront les citoyens non seulement à se savoir égaux mais également à se sentir égaux, en cultivant par exemple la vertu de tolérance, qui constitue une intériorisation de la valeur d'égalitée ${ }^{56}$.

Rawls répond ici en un sens à certaines objections républicaines, d'inspiration tocquevillienne. Il montre que le libéralisme politique n'est pas si démuni que certains républicains l'affirment, et qu'il est capable de produire sa propre stabilité.

Néanmoins, des républicains comme Laborde pourront considérer que cela ne suffit pas à résoudre le problème spécifique qu'ils pointent, à savoir le problème des conditions de formation des préférences. Cultiver la vertu de tolérance peut amener les jeunes à accepter les convictions des

55. Rawls, Libéralisme politique, p. 239. Je souligne.

56. Rawls n'indique pas néanmoins quel type de politiques éducatives concrètes sont susceptibles de produire de tels effets. On trouvera à mon avis matière à prolonger cette réflexion dans Maclure et Taylor, Laïcité et liberté de conscience, notamment p. 55-63. Maclure et Taylor y développent ce qu'on peut appeler l'hypothèse du contact, selon laquelle la fréquentation, dès le plus jeune âge, de la diversité culturelle et religieuse serait susceptible de renforcer la tolérance. 
autres, malgré le dégoût spontané qu'ils peuvent éventuellement ressentir, mais cela suffit-il à former leur esprit critique et à les rendre capables de mettre à distance leurs propres options existentielles? Cela suffit-il à les préserver des mécanismes de domination? Si ce n'est pas le cas, on considérera peut-être qu'il faut continuer à se tourner vers le républicanisme, qu'on concevra alors comme une solution de rechange au libéralisme.

Mais le républicanisme doit-il être compris comme une solution de rechange au libéralisme, solution qui parvient à éviter les écueils du libéralisme grâce à un perfectionnisme assumé ? Cette lecture n'est pas sans poser un certain nombre de difficultés. Pour terminer, je confronterai deux interprétations possibles du républicanisme critique de Laborde: une interprétation perfectionniste et une interprétation neutraliste.

\subsection{Un républicanisme critique perfectionniste}

Les républicains considèrent souvent que c'est le perfectionnisme assumé du républicanisme qui lui permet d'être une option de remplacement attractive au libéralisme politique. Ainsi Laborde, à l'instar de Philip Pettit, assume "l'idée que la théorie républicaine bien comprise prom[eut] une valeur particulière: celle de la non-domination ${ }^{57}{ }^{»}$. Elle considère aussi que c'est ce parti-pris en faveur de la non-domination qui permet au républicanisme critique d'être attentif aux conditions dans lesquelles les individus forment leurs préférences, alors que les libéraux sont contraints de s'en tenir aux préférences existantes.

Mais si Laborde assume une forme de perfectionnisme, il est clair néanmoins que son perfectionnisme n'est pas le perfectionnisme du républicanisme classique. En premier lieu, la valeur promue n'est pas la même. Le libéralisme classique promouvait la liberté entendue comme liberté de pensée et autonomie rationnelle. Laborde récuse ce perfectionnisme étroit qui considère qu'un usage permanent de la raison critique est la condition de possibilité de la vie bonne. Selon elle, le républicanisme critique doit au contraire admettre qu'il existe plusieurs façons de mener une vie bonne. Le républicanisme est bien plus procédural que le républicanisme classique. Ce qui fait la qualité d'un mode de vie, c'est n'est pas tant son contenu que les conditions dans lesquelles il est adopté. Ces conditions doivent être celles de la non-domination. En outre, on l'a vu, le perfectionnisme critique rejette l'usage de la coercition. L'État ne peut prétendre produire de la liberté en faisant usage de la coercition.

Reste que tant que le républicanisme critique se présente comme une conception perfectionniste, il demeure vulnérable à l'égard d'une objection sérieuse au vu de laquelle il n'est plus du tout évident qu'il soit plus attractif que le libéralisme politique. L'objection est simple et peut être formulée 
comme suit: pourquoi accepter que l'État promeuve cette valeur qu'est la non-domination? Pourquoi cette valeur plutôt qu'une autre? Quelle est la justification de la promotion de la non-domination?

Il n'est pas du tout évident qu'un républicanisme perfectionniste puisse fournir une réponse à ces questions. La valeur valorisée y demeure un axiome ininterrogé. Il est possible que, pour des raisons normatives, on éprouve spontanément de la sympathie à l'égard de cette valeur qu'est la non-domination. Néanmoins, d'un point de vue théorique, le fait qu'elle demeure injustifiée est problématique. La préférence pour la non-domination semble arbitraire. Elle nous laisse sans réponse face à ceux qui assumeront un perfectionnisme promouvant des valeurs pour lesquelles nous avons moins de sympathie.

Néanmoins, le républicanisme critique de Laborde peut, à mon avis, dépasser cette difficulté. Mais il faut alors renoncer à en faire une lecture perfectionniste. Il faut l'interpréter comme une position neutraliste.

\subsection{Un républicanisme critique neutraliste}

Peut-on lire le républicanisme critique autrement que comme une position perfectionniste? Certains éléments me semblent accréditer une telle lecture. Dans Critical Republicanism, livre écrit en anglais dont Français encore un effort pour être républicain! est une synthèse, Laborde écrit:

L'autonomie que les républicains critiques promeuvent ressemble plutôt à une capabilité [...]. Conçue comme une capabilité minimale, l'autonomie ne doit plus être comprise comme un bien intrinsèque mais comme un bien instrumental. C'est néanmoins un bien premier. C'est un ingrédient essentiel pour vivre une vie réussie, une vie qui est bonne en elle-même, mais ce n'est pas nécessairement une composante de ce succès ${ }^{58}$.

Pour parler de la valeur que le républicanisme critique promeut, Laborde recourt à la fois au vocabulaire des capabilités et au vocabulaire des biens premiers. Ces deux concepts ont pourtant des origines théoriques distinctes $^{59}$. Je me concentrerai pour ma part sur le vocabulaire des biens premiers.

C’est Rawls qui, dans Théorie de la justice, a forgé le concept de bien premier $^{60}$. Les biens premiers sont ces biens «que tout homme rationnel est supposé désirer ", parce qu'ils sont "utiles, quel que soit notre projet de vie rationnel ${ }^{61} »$. Les biens premiers indiquent l'existence d'une « ressemblance

58. Laborde, Critical Republicanism, p. I 55. Je traduis.

59. Le concept de capabilité a été forgé par Amartya Sen, Ethique et économie. Cette approche a ensuite été développée par Martha Nussbaum, Capabilités.

60. Pour une définition du concept de bien premier, on se reportera en particulier à Rawls, Théorie de la justice, p. 93, p. I22-I23.

61. Rawls, Théorie de la justice, p. 93. 
partielle dans la structure des conceptions du bien ${ }^{62}$ ». Ce sont des biens que nous désirons - quoi que nous désirions par ailleurs. Ce sont des biens qui favorisent la réalisation de toute conception de la vie bonne. Rawls distingue les biens premiers naturels des biens premiers sociaux. La santé, l'intelligence ou l'imagination figurent parmi les premiers. Les droits et libertés, les possibilités sociales offertes aux individus, les revenus et la richesse parmi les seconds.

Mais quel rapport la théorie de la justice comme équité entretient-elle avec les biens premiers? Quel rapport cette théorie libérale tenue à la neutralité vis-à-vis des doctrines compréhensives raisonnables controversées entretient-elle avec les biens premiers? Il est essentiel de comprendre que, pour Rawls, de la même façon que le libéralisme politique n'a pas à être neutre vis-à-vis des valeurs de liberté et d'égalité, la TJE n'a pas à être neutre vis-à-vis des biens premiers. Dès Théorie de la justice, Rawls entend définir des principes de justice sans dériver le juste du bien. Sous le voile d'ignorance, les partenaires ignorent à quelle conception particulière du bien ils adhéreront. Néanmoins, pour parvenir à des principes de justice, une théorie minimale du bien est nécessaire. Les partenaires doivent savoir quels sont leurs intérêts fondamentaux, intérêts qu'ils vont s'appliquer à protéger. La TJE n'a pas à être neutre vis-à-vis des biens premiers. Puisque ces biens ne sont pas des biens controversés, l'État libéral peut légitimement les promouvoir sans se transformer en État perfectionniste.

Mais revenons au républicanisme critique. Quelles sont les conséquences de cette façon de concevoir la non-domination? Que se passe-t-il, d'un point de vue théorique, si la non-domination peut être comprise comme un bien premier? Les conséquences théoriques sont décisives. Si la nondomination peut être considérée comme un bien premier, alors elle peut être promue sans que la théorie républicaine ne doive être considérée comme une théorie perfectionniste. Pour le dire autrement, affirmer que le républicanisme critique promeut la non-domination et dans le même temps que la non-domination est un bien premier, c'est affirmer que le républicanisme critique peut être compris comme une position neutraliste plutôt que comme une position perfectionniste.

Quels sont les avantages et les éventuelles difficultés de cette lecture? L'avantage considérable d'une interprétation neutraliste du républicanisme critique, c'est qu'elle apporte une solution au problème de la valorisation arbitraire pointé plus haut. Si la non-domination est un bien premier, la valorisation de la non-domination peut être justifiée. Plus précisément, elle peut l'être de deux façons différentes et complémentaires.

Premièrement, la non-domination est d'abord une interprétation de cette valeur générique qu'est la liberté. Or le libéralisme n'a pas à être neutre vis-à-vis de ces valeurs politiques fondamentales que sont la liberté et l'éga-

62. Rawls, Libéralisme politique, p. 223. 
lité. Ces valeurs peuvent, conformément au "principe libéral de légitimité », légitimement être invoquées lorsqu'il s'agit de justifier la loi. Ces valeurs sont en effet des valeurs communes, que l'ensemble des citoyens doivent reconnaître. Ainsi, puisqu'il est légitime d'accorder de la valeur à la liberté, il est également légitime de valoriser la non-domination dans la mesure où la non-domination n'est rien d'autre qu'une interprétation possible de la liberté.

Deuxièmement, la non-domination peut être justifiée en tant qu'interprétation particulière de la liberté. Pour le comprendre, il faut se rappeler que si, dans un cadre libéral-neutraliste, la valorisation de la liberté et de l'égalité est justifiée, ces valeurs possèdent de prime abord une forme d'indétermination. La liberté et l'égalité ont en premier lieu une forme générale. Elles doivent néanmoins nécessairement faire l'objet d'une interprétation spécifique pour pouvoir engendrer des principes de justice précis. C'est notamment la raison pour laquelle Rawls assume une interprétation particulière de ces valeurs que sont la liberté et l'égalité. C'est également pour cette raison que Rawls affirme régulièrement que la TJE est une "forme", parmi d'autres, "du libéralisme politique ${ }^{63}$ ». La TJE propose une interprétation, parmi d'autres interprétations possibles, des valeurs de liberté et d'égalité.

Dans cette perspective, le républicanisme critique peut être compris comme une interprétation concurrente des valeurs de liberté et d'égalité. Le républicanisme critique serait, en particulier, cette théorie qui rejetterait l'interprétation rawlsienne de la liberté et qui lui opposerait une autre interprétation: la liberté comme non-domination. Le républicanisme critique se caractériserait par l'affirmation selon laquelle il faut abandonner l'interprétation rawlsienne de la liberté, qui présente un certain nombre de défauts, et notamment une naïveté excessive vis-à-vis des conditions de formation de nos préférences, et lui préférer la liberté comme non-domination qui constitue une meilleure interprétation de la liberté.

Si l'on accepte cette lecture, il serait tout à fait possible, pour les républicains, de justifier leur interprétation de la liberté en reprenant à leur compte les outils épistémologiques développés par Rawls. Il leur suffirait notamment de montrer que leur interprétation de la liberté réalise mieux l'équilibre réfléchi que l'interprétation rawlsienne. Il leur faudrait simplement montrer que leur interprétation de la liberté parvient, mieux que l'interprétation rawlsienne, à mettre en cohérence les intuitions normatives et non normatives auxquelles, après réflexion, nous ne souhaitons pas renoncer. Si leur démonstration était convaincante, ils disposeraient, de l'aveu même des libéraux, d'une justification de leur conception de la liberté.

Malgré les bénéfices théoriques inhérents à cette interprétation neutraliste du républicanisme, certains républicains pourraient néanmoins répu-

63. Rawls, Libéralisme politique, p. 250. 
gner à l'accepter. Il faut en effet insister sur le fait que cette interprétation nous contraint à réviser la façon dont le républicanisme est très souvent présenté. Le républicanisme ne peut plus être conçu comme une solution de rechange au libéralisme politique. Le républicanisme ne peut plus être conçu comme cette théorie parfaitement distincte, qui permettrait de sortir des impasses du libéralisme politique. Il n'est plus qu'une forme de libéralisme politique. Il se distingue sur plusieurs points de cette variante qu'est la TJE, mais il trouve tout à fait sa place dans l'architecture générale du libéralisme politique.

Cécile Laborde accepte à mon avis aujourd'hui cette façon d'envisager les choses. Elle considère que le type de républicanisme qu'elle défend s'intègre à cette grande famille du libéralisme politique. Mais si le républicanisme critique doit être considéré comme une théorie neutraliste, il demeure essentiel de savoir comment il convient d'interpréter l'exigence de neutralité et le concept de raison publique. C'est d'ailleurs l'une des questions que Laborde discute dans son dernier livre, Liberalism's Religion ${ }^{64}$.

\section{Conclusion}

Faut-il, comme on le fait très souvent, interpréter le républicanisme comme une option de rechange au libéralisme politique? Le républicanisme parvient-il, parce qu'il est perfectionniste, à dépasser le libéralisme politique qui, en raison de son engagement neutraliste, est condamné à toute une série d'impasses? Telles sont les questions auxquelles j'ai cherché à apporter des éléments de réponse en m'appuyant sur le cas de la liberté de conscience. Je considère que, s'il existe bien des différences importantes entre le libéralisme politique rawlsien et un républicanisme revisité comme le républicanisme critique de Cécile Laborde, l'opposition entre neutralisme et perfectionnisme n'est pas la plus pertinente pour en prendre la mesure. Ce type de républicanisme gagne en solidité théorique à s'affirmer comme une position neutraliste. Il ne peut dès lors plus néanmoins se positionner comme une solution de rechange.

\section{Bibliographie}

Audier, Serge. Les théories de la république, Paris, La Découverte, 2004. Barry, Brian. Justice as Impartiality, Oxford, Clarendon Press, 1995.

Baudoin, Jean et Philippe Portier (dir.). La laïcité, valeur d'aujourd'bui? Contestations et renégociations du modèle français, Rennes, Presses universitaires de Rennes, 200I.

Baubérot, Jean. La morale lä́que contre l'ordre moral, Paris, Seuil, I997.

—_. "La laïcité comme pacte laïque» in Baudoin et Portier (dir.), La laïcité, valeur d'aujourd'bui?, Rennes, Presses universitaires de Rennes, $200 \mathrm{I}$. 
- Les sept laïcités françaises, Paris, Éditions de la Maison des sciences de l'homme, 20I 5 .

Berten, André, Pablo Da Silveira et Hervé Pourtois. Libéraux et communautariens, Paris, PUF, I997.

Darwall, Stephen, Allan Gibbard et Peter Railton. "Towards Fin de siecle Ethics: Some Trends ", The Philosophical Reviews, IоI, I, I992, p. II 5-189.

Desmons, Ophélie. "Pourquoi la théorie politique normative doit s'intéresser à la métaéthique: le cas de la théorie de la justice et du constructivisme ", Implications philosophiques, 2013.

Dilhac, Marc-Antoine. La tolérance, un risque pour la démocratie?, Paris, Vrin, $20 \mathrm{I} 4$.

Dunn, John. La pensée politique de John Locke, Paris, PUF, I99I.

Dworkin, Ronald. Taking Right Seriously, Cambridge, Mass., Harvard University Press, I977.

—. "Le libéralisme », in Berten, Da Silveira, Pourtois, Libéraux et communautariens, p. 5 I-86.

Kintzler, Catherine. «Aux fondements de la laïcité scolaire. Essai de décomposition raisonnée du concept de laïcité », Les Temps modernes, 527, p. 82-90.

—. La République en question, Paris, Minerve, I996.

- Qu'est-ce que la laïcité?, Paris, Vrin, 2008.

- Penser la laïcité, Paris, Minerve, 20I4.

Laborde, Cécile. Critical Republicanism, The Hijab Controversy and Political Philosophy, Oxford, Oxford University Press, 2008.

—. Français, encore un effort pour être républicains!, Paris, Seuil, 2010.

—. Liberalism's Religion, Cambridge, Harvard University Press, 2017.

Larmore, Charles. Patterns of Moral Complexity, Cambridge, Cambridge University Press, 1987.

- Modernité et morale, Paris, PUF, I993.

Locke, John, Lettre sur la tolérance, Paris, GF, 2007.

Maclure, Jocelyn et Charles Taylor. Laïcité et liberté de conscience, Paris, La Découverte, 20 Iо.

Nussbaum, Martha. Capabilités. Comment créer les conditions d'un monde plus juste?, Paris, Flammarion, $20 \mathrm{I} 2$.

- Les religions face à l'intolérance. Vaincre la politique de la peur, Paris, Flammarion, 20I3.

Pettit, Philip. Républicanisme: Une théorie de la liberté et du gouvernement, Paris, Gallimard, 2004.

- On the People's Term, A Republican Theory and Model of Democracy, Cambridge, Cambridge University Press, 2012.

Pocock, John Greville Agard. Le moment machiavélien: La pensée politique florentine et la tradition républicaine atlantique, Paris, PUF, I998.

- Political Thought and History, Essays on Theory and Method, Cambridge, Cambridge University Press, 2009.

Rawls, John. Théorie de la justice, Paris, Seuil, I987.

—. "Le constructivisme kantien dans la théorie morale ", in Justice et démocratie, p. 7I-I 52 .

- Justice et démocratie, Paris, Seuil, I993.

—. Libéralisme politique, Paris, PUF, I995. 
- La justice comme équité: Une reformulation de la théorie de la justice, Paris, La Découverte, 2008.

Renaut, Alain. Qu'est-ce qu'un peuple libre? Libéralisme ou républicanisme, Paris, Cerf, 2005.

Rousseau, Jean-Jacques. Du contrat social, Paris, Flammarion, 200 I.

Sandel, Michael. "La république procédurale et le moi désengagé », in Berten, Da Silveira et Pourtois, Libéraux et communautariens, p. 25 5-274.

- Le libéralisme et les limites de la justice, Paris, Seuil, I999.

Sen, Amartya. Éthique et économie, Paris, PUF, 2008.

Skinner, Quentin. Machiavel, Seuil, I989.

—. La liberté avant le libéralisme, Seuil, 2000.

. Hobbes et la conception républicaine de la liberté, Paris, Albin Michel, 2009.

Spitz, Jean-Fabien. Le moment républicain en France, Paris, Gallimard, 2005.

—. Philip Pettit: Le républicanisme, Paris, Michalon, 2010.

Taylor, Charles. Malaise de la modernité, Paris, Cerf, I994.

"Quiproquos et malentendus: le débat communautariens-libéraux », in Berten, Da Silveira, et Pourtois, Libéraux et communautariens, p. 87-I20.

- Multiculturalisme: Différence et démocratie, Paris, Flammarion, 2009.

Tocqueville, Alexis de. De la démocratie en Amérique, Paris, GF, I98I. 